\title{
Iterative Learning for Reliable Underwater Link Adaptation (Student Abstract)
}

\author{
Junghun Byun, ${ }^{1}$ Yong-Ho Cho, ${ }^{2}$ Tae-Ho Im, ${ }^{2}$ Hak-Lim Ko, ${ }^{2}$ Kyung-Seop Shin, ${ }^{3}$ Ohyun Jo ${ }^{1, *}$ \\ ${ }^{1}$ Chungbuk National University, 28644, Cheongju, Republic of Korea \\ ${ }^{2}$ Hoseo University, 31499, Asan, Republic of Korea \\ ${ }^{3}$ Semyung University, 27136, Jecheon, Repulbic of Korea \\ *Corresponding author (ohyunjo@chungbuk.ac.kr)
}

\begin{abstract}
This paper describes an iterative learning framework consisting of multi-layer prediction processes for underwater link adaptation. To obtain a dataset in real underwater environments, we implemented OFDM (Orthogonal Frequency Division Multiplexing)-based acoustic communications testbeds for the first time. Actual underwater data measured in Yellow Sea, South Korea, were used for training the iterative learning model. Remarkably, the iterative learning model achieves up to $25 \%$ performance improvement over the conventional benchmark model.
\end{abstract}

\section{Introduction}

Underwater Wireless Sensor Networks (UWSNs) have been receiving considerable attention recently for various applications such as marine industry, climate, oceanic disaster prevention, military, etc. Recent researches in UWSN are mainly focused on the problems due to limited power supply and propagation delay(Chen et al. 2014). Adaptive Modulation and Coding (AMC) is a link adaptation technique that provides the most suitable transmit parameters out of predefined MCS levels based on channel status. By using AMC, we can utilize the resource more efficiently mitigating unintended power consumption. Conventionally, AMC utilizes the strong negative correlation between Signal to Noise Ratio (SNR) and Bit Error Rate (BER). However, in UWSNs, the conventional SNR-based method can not be applied unfortunately because there are a number of external factors which affect BER performance in the underwater environment. To tackle this challenge, machine learning techniques can be a good solution because they can provide appropriate parameters by considering various external environment parameters collectively(Pelekanakis et al. 2016).

In this paper, an underwater dataset generated by experiments in real environments is introduced, and an iterative learning framework based on Multiple Linear Regression (MLR) and Multi-Layer Perceptron (MLP) is also proposed. Performance of the proposed iterative scheme is evaluated via practical experiments and intensive analysis under real underwater environments.

Copyright (C) 2020, Association for the Advancement of Artificial Intelligence (www.aaai.org). All rights reserved.

\section{Iterative Learning Framework for Underwater Link Adaptation}

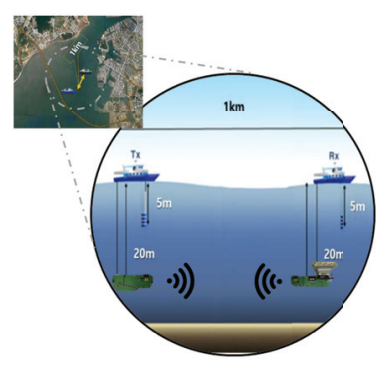

(a)

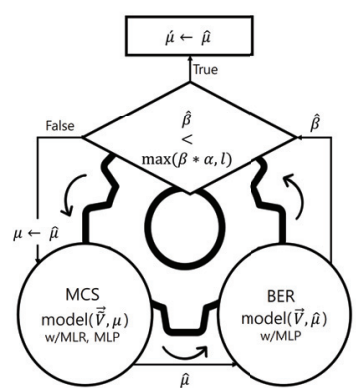

(b)
Figure 1: (a) Real underwater environments and testbeds, Gulf of Incheon, South Korea. (b) Iterative learning framework based on MLR and MLP.

Table 1: Symbol Description

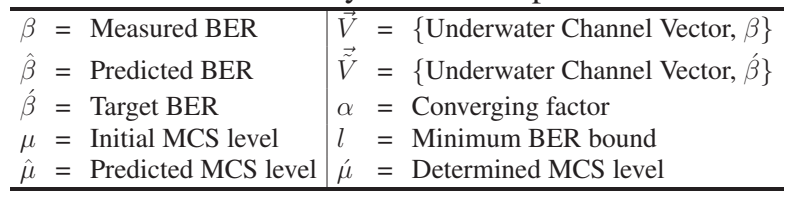

\section{Underwater Dataset Generation}

The training of the model uses underwater link quality data which is collected under real underwater environments near Gulf of Incheon, South Korea, as shown in Figure 1 (a). The total number of samples in the dataset is 10151, and each data sample contains the measured Underwater Channel Vector which is the set of underwater channel parameters including SNR, CINR (Carrier to Interference and Noise Ratio), delay spread, maximum delay component, Doppler frequency shift, coded BER, and uncoded BER. Based on the relevance of the these practically measured parameters, the most suitable MCS can be determined. 


\section{Iterative Learning Model}

Conventionally, the MCS level is determined depending on SNR which has strong correlation with BER. However, based on our hands-on experience obtained from oceanic field tests, it is found that the single SNR parameter is not strongly correlated with the BER performance in underwater environment. Thus, the proposed iterative learning method takes into account the multiple parameters collectively to predict MCS and its BER more accurately by using machine learning. The iterative algorithm consists of three steps as shown in the Figure 1 (b).

Input vector for the iterative learning model, $\vec{V}$, can be obtained from the reception and analysis of pilot signals sent by the peer sensor node. MCS model finds $\hat{\mu}$ using $\overrightarrow{\tilde{V}}, \mu$. Then, BER model finds $\hat{\beta}$ using $\vec{V}$ and $\hat{\mu}$, and checks if $\hat{\beta}$ meets the criteria which is defined as $\hat{\beta}<\max (\beta * \alpha, l)$. If the criterion is not satisfied, $\mu$ is updated to $\hat{\mu}$ and the iterative learning processes are repeated. Otherwise, the MCS level will be decided as $\hat{\mu}$ for next transmission.

\section{Performance Evaluation}

We compared three machine learning based models which are MLR model, MLP model, and SNR-based model, for performance evaluation. MCS model and BER model have been verified by MLR model, MLP model, and SNR-based model, respectively. SNR-based model which is regarded as the conventional scheme uses linear regression to determine the MCS level by using a single parameter (SNR) only. The dataset is splitted into training set and validation set. The ratio is $8: 2$. These three models were trained using the same dataset obtained from our implementations and experiments. And the predicted data rate for each case would be compared to the data rate configured in the dataset, which is randomly selected. In the experiments, $\hat{\beta}$ was set to $0.3, \alpha$ to 0.5 and $l$ to 0.1 . Figure 2 shows the results predicted by three models. MLP model predicted higher data rate while satisfying the BER requirements.

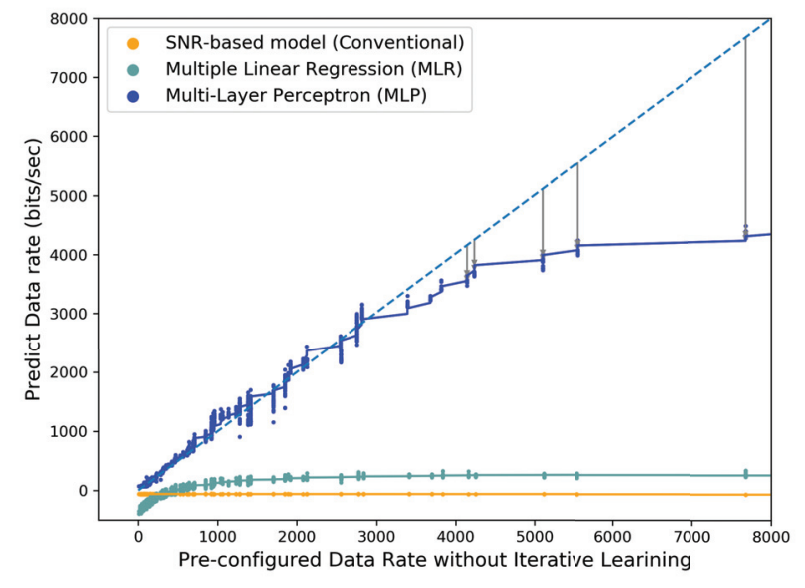

Figure 2: Pre-configured data rate w/o Iterative Learning vs. Properly predicted data rate.
SNR-based model predicted very low data rate regardless of the pre-configured data rate. This means that SNR alone could not decide the adequate MCS level. The measured BER with the pre-configured data rate without iterative learning was very high, that means, randomly selected MCS may result in a lot of transmission failures. MLR model and MLP model are reducing data rate regularly while the target BER criterion is properly satisfied.

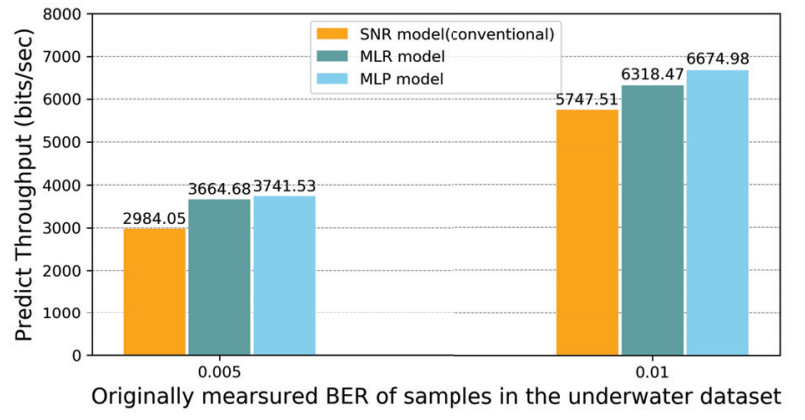

Figure 3: Performance comparison of SNR model, MLR model, and MLP model.

Figure 3 shows the results of applying each model to the validation data. The measured BER of sample data without iterative learning processes was 0.005 and 0.01 , respectively. Total throughput is expected to increase up to $25 \%$ by using the iterative learning model. Generally, MLP model outperforms MLR model. On the other hand, the computational complexity of MLP is supposed to be higher than that of MLR model.

\section{Conclusion}

Conclusively, this paper proposed and evaluated an iterative learning model for underwater link adaptation. Through the practical performance evaluations under real environments, we are convinced that the iterative learning can effectively increase the total throughput for UWSNs. For further enhancements, we are studying with additional environmental data such as water temperature, currents, salinity, etc.

\section{Acknowledgment}

This research was a part of the project titled 'Development of Distributed Underwater Monitoring and Control Networks', funded by the Ministry of Oceans and Fisheries, Korea. And this work was supported by the National Research Foundation of Korea (NRF) grant funded by the Korea government (MSIT) (No. NRF-2018R1C1B5045013).

\section{References}

Chen, K.; Ma, M.; Cheng, E.; Yuan, F.; and Su, W. 2014. A survey on MAC protocols for underwater wireless sensor networks. IEEE Communications Surveys \& Tutorials 16(3):1433-1447.

Pelekanakis, K.; Cazzanti, L.; Zappa, G.; and Alves, J. 2016. Decision tree-based adaptive modulation for underwater acoustic communications. In 2016 IEEE Third Underwater Communications and Networking Conference, 1-5. IEEE. 